\title{
The ESO UVES Advanced Data Products Quasar Sample - V. Identifying the Galaxy Counterpart to the sub-Damped Ly $\alpha$ System towards Q 2239-2949^
}

\author{
Tayyaba Zafar ${ }^{1,2} \dagger$, Palle Møller ${ }^{2}$, Céline Péroux ${ }^{3}$, Samuel Quiret ${ }^{3}$, \\ Johan P. U. Fynbo ${ }^{4}$, Cédric Ledoux ${ }^{5}$, and Jean-Michel Deharveng ${ }^{3}$ \\ 1 Australian Astronomical Observatory, PO Box 915, North Ryde, NSW 1670, Australia. \\ 2 European Southern Observatory, Karl-Schwarzschild-Strasse 2, 85748, Garching, Germany. \\ 3 Aix Marseille Université, CNRS, LAM (Laboratoire d'Astrophysique de Marseille) UMR 7326, 13388, Marseille, France. \\ 4 Dark Cosmology Centre, Niels Bohr Institute, University of Copenhagen, Juliane Maries Vej 30, DK-2100 Copenhagen, Denmark. \\ 5 European Southern Observatory, Alonso de Córdova 3107, Vitacura, Casilla 19001, Santiago 19, Chile.
}

3 August 2018

\begin{abstract}
Gas flows in and out of galaxies are one of the key unknowns in todays' galaxy evolution studies. Because gas flows carry mass, energy and metals, they are believed to be closely connected to the star formation history of galaxies. Most of these processes take place in the circum-galactic medium (CGM) which remains challenging to observe in emission. A powerful tool to study the CGM gas is offered by combining observations of the gas traced by absorption lines in quasar spectra with detection of the stellar component of the same absorbing-galaxy. To this end, we have targeted the $z_{\mathrm{abs}}=1.825$ sub-Damped Ly $\alpha$ absorber (sub-DLA) towards the $z_{\mathrm{em}}=2.102$ quasar 2dF J 223941.8-294955 (hereafter Q 2239-2949) with the ESO VLT/X-Shooter spectrograph. Our aim is to investigate the relation between its properties in emission and in absorption. The derived metallicity of the sub-DLA with $\log N(\mathrm{HI})$ $=19.84 \pm 0.14 \mathrm{~cm}^{-2}$ is $[\mathrm{M} / \mathrm{H}]>-0.75$. Using the Voigt profile optical depth method, we measure $\Delta v_{90}(\mathrm{Fe}$ II $)=64 \mathrm{~km} \mathrm{~s}^{-1}$. The sub-DLA galaxy counterpart is located at an impact parameter of $22^{\prime \prime} 4 \pm 00^{\prime \prime} 2(20.8 \pm 1.7 \mathrm{kpc}$ at $z=1.825)$. We have detected Ly $\alpha$ and marginal [O II] emissions. The mean measured flux of the Ly $\alpha$ line is $F_{\mathrm{Ly} \alpha} \sim 5.7 \times 10^{-18}$ $\operatorname{erg~s}^{-1} \mathrm{~cm}^{-2} \AA^{-1}$, corresponding to a dust uncorrected SFR of $\sim 0.13 \mathrm{M}_{\odot} \mathrm{yr}^{-1}$.
\end{abstract}

Key words: Galaxies: formation - galaxies: evolution - galaxies: abundances - galaxies: ISM - quasars: absorption lines - intergalactic medium

\section{INTRODUCTION}

One of the key unknowns in the study of galaxy evolution is how galaxies acquire their gas and how they exchange this gas with their surroundings. Since gas, stars, and metals are intimately connected, gas flows affect the history of star formation and chemical enrichment in galaxies. Therefore study of the circum-galactic medium (CGM, extending over $\sim 300 \mathrm{kpc}$ around galaxies; Shull et al. 2014) is crucial for understanding both the inflows of gas accreted onto galax-

\footnotetext{
* Based on observations collected during programmes ESO 069.A-0586, 077.A-0714 and 091.A-0246 at the European Southern Observatory with UVES and X-Shooter on the $8.2 \mathrm{~m}$ telescopes operated at the Paranal Observatory, Chile.

$\dagger$ e-mail:tayyaba.zafar@aao.gov.au
}

ies and the outflows carrying away the energy and metals generated inside galaxies (Kereš et al. 2012; Ceverino et al. 2015). A powerful tool to study the CGM gas is offered by absorption lines in quasar spectra. Indeed, quasar sightlines provide a wealth of information about the CGM of galaxies through the analysis of the intervening absorption line systems. The detection of damped Ly $\alpha$ systems (DLAs; $\log \mathrm{N}(\mathrm{HI}) \geqslant 20.3 \mathrm{~cm}^{-2}$ ) and sub-damped Ly $\alpha$ systems (subDLAs; $\left.19.0 \leqslant \log \mathrm{N}(\mathrm{H} \mathrm{I})<20.3 \mathrm{~cm}^{-2}\right)$ in absorption against bright background quasars is in principle not limited by the luminosity of the associated galaxies but depends on the cross-section of the neutral hydrogen gas (e.g., Wolfe et al. 1986). It is now established that gas accretion on global scales is required to reconcile the lack of evolution in the neutral gas mass over large cosmological times with the observed SFR density evolution (e.g., Noterdaeme et al. 2009; 
Zafar et al. 2013a; Sánchez-Ramírez et al. 2015). The DLAs and sub-DLAs are therefore excellent laboratories to study metals, molecules, dust, and atomic gas, an indirect indicator of star formation (e.g., Krumholz 2012; Zafar et al. 2013a).

However, the background quasar probes only a single sightline through the galaxy, and it is difficult to study gas flows in the underlying galaxy as a whole from the gas along a single sightline. To establish the connection of the CGM gas probed by the quasar sightline to the underlying galaxy, it is essential to complement the absorption spectroscopy with studies of the absorbing galaxy in emission, and determine the kinematics and metallicity of the emission-line gas. To reach this goal the first step is to identify DLA host-galaxies in emission in large enough numbers to be able to perform statistical studies (e.g. Péroux et al. 2011; Krogager et al. 2012; Peroux et al. 2016). Until recently, deep, high spatial resolution images were obtained to identify potential DLA galaxies nearby the quasar line of sight (Warren et al. 2001), followed by spectroscopic observations to verify that the galaxies lie at the same redshifts as the DLAs (Weatherley et al. 2005). Today, improved selection strategies using long-slit triangulations (e.g., Fynbo et al. 2011; Noterdaeme et al. 2012; Fvnbo et al. 2013; Krogager et al. 2013) or integral field units (e.g., Bouché et al. 2007; Péroux et al. 2012, 2013) have led to several new spectroscopic detections of DLA galaxies in emission.

In parallel, the discovery that high- $z$ DLA galaxies obey luminosity-metallicity and velocity-metallicity relations with similar slopes as in the local Universe, Møller et al. (2004); Ledoux et al. (2006) and Neeleman et al. (2013) allows one to relate flux limited galaxy samples and DLA selected samples (Møller et al. 2013; Christensen et al. 2014). Establishing this connection reinforces the possibility of quasar absorbers to trace the evolution of faint galaxies even back to redshifts where they are too faint to be seen in emission (Fvnbo et al. 2008). It is therefore of high importance to extend the sample of DLA galaxies seen in emission, and to extend the parameter space of detections (e.g. to lower H I column densities (sub-DLAs) and to lower metallicities) to better probe the faint-end of the galaxy luminosity function.

Here, we report the detection of a new absorbing-galaxy in the field of Q 2239-2949. The quasar $\left(z_{\mathrm{em}}=2.102, B=\right.$ $19.31 \mathrm{mag})$ was first discovered in the 2-degree Field (2dF) survey at the Anglo Australian Telescope Croom et al. 2001). Using ESO/UV and Visual Echelle Spectrograph (UVES) archival data, Zafar et al. (2013b) report the detection of Ly $\alpha$ flux in emission in the trough of the subDLA at $z=1.825$ in the sightline towards Q 2239-2949. In this paper, we present new X-Shooter observations of the absorbing-galaxy detected in emission which allows one to characterise the properties of its stellar components as well as a complete analysis of archival UVES data to extract fundamental parameters of the neutral gas seen in absorption.

We adopt the standard $\Lambda$ CDM cosmology with the cosmological parameters $\Omega_{\Lambda}=0.73, \Omega_{m}=0.27$, and $H_{0}=70$ $\mathrm{km} \mathrm{s}^{-1} \mathrm{Mpc}^{-1}$.

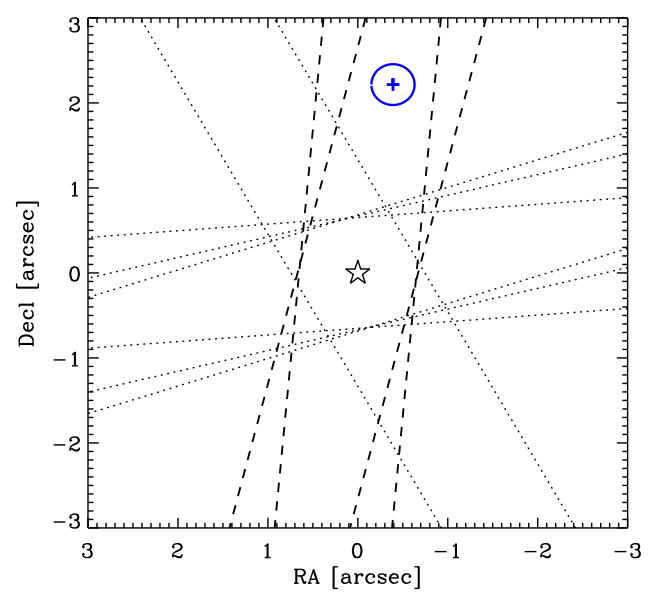

Figure 1. Slit positions used for the X-Shooter observations of Q 2239-2949 (marked by a star). The dashed lines show the slit positions $\mathrm{PA}=-14^{\circ} \&-5^{\circ}$, where we detect emission line flux from the sub-DLA galaxy. The probable position of the galaxy is marked by a cross and a blue circle at an impact parameter of 2 '. 4 . At other slit positions (dotted lines) no emission line flux is detected.

\section{OBSERVATIONS AND DATA REDUCTION}

\subsection{UVES spectroscopy}

The quasar Q 2239-2949 was first observed with UVES (Dekker et al. 2000) with three different settings as part of the tomography of the intergalactic medium (IGM) programs (69.A-0586(A) PI: Cristiani, 077.A-0714(A) PI: D'Odorico). The EUADP (ESO UVES Advanced Data Products) archival data (Zafar et al. 2013b. a) did not include all the data taken on that object, therefore, we retrieved the additionally available raw data and reduced it using the UVES pipeline 5.4.0 (Ballester et al. 2000). The complete log of observations is provided in Table 1

The raw frames are corrected for bias level, flat-field, and rectified in wavelength space using a wavelength solution obtained from calibration frames. The orders are then merged and $1 \mathrm{D}$ spectra are extracted from the merged $2 \mathrm{D}$ spectra. The resulting spectrum ranges from 3024 to $10433 \AA$. The spectra from different settings are corrected to the vacuum heliocentric reference system and merged into a single spectrum following the method described by Zafar et al. (2013b). The merged quasar continuum is then normalised by fitting a spline function passing through spectral chunks of $200 \AA$ apparently devoid of absorption lines.

\subsection{X-Shooter spectroscopy}

During our inspection of the initial UVES data, we noticed that Ly $\alpha$ emission flux from the absorbing galaxy is detected in the trough of the absorption in individual UVES spectra (see Fig. 20 of Zafar et al. 2013b. In order to precisely measure the position on sky, impact parameter as well as the emission properties of the galaxy traced by itsLy $\alpha$ emission, we then re-observed Q 2239-2949 with VLT/X-Shooter (Vernet et al. 2011) using six different "Observation Blocks" under program ID 091.A-0246(A) (PI: Zafar; observing log provided in Table 1). The exposure times were $1 \mathrm{~h}$ each. The 
Table 1. Log of spectroscopic observations of Q 2239-2949 from UVES archival and X-Shooter PI time.

\begin{tabular}{|c|c|c|c|c|c|c|c|c|c|}
\hline Date & $\begin{array}{l}\mathrm{T}_{\exp } \\
(\mathrm{sec})\end{array}$ & Instrument & Settings & $\begin{array}{c}\text { Slit } \\
\text { width }\end{array}$ & $\begin{array}{c}\text { PA } \\
(\operatorname{deg})\end{array}$ & $\begin{array}{l}\text { Resolving power } \\
\text { for each arm }\end{array}$ & Prog. ID & Seeing & Airmass \\
\hline 17 Jun, 2002 & $1512 \times 2$ & UVES & BLUE437, RED860 & $1.1^{\prime \prime}$ & & 39090,42310 & 69.A-0586(A) & $1.0^{\prime \prime}$ & 1.1 \\
\hline 12 Aug, 2002 & $3065 \times 3$ & UVES & BLUE346, RED580 & $1.1^{\prime \prime}$ & & 39090,37820 & 69.A-0586(A) & $0.9^{\prime \prime}$ & 1.0 \\
\hline 27 May, 2006 & 3000 & UVES & BLUE346, RED580 & $1.0^{\prime \prime}$ & & 40970,42310 & 077.A-0714(A) & $1.2^{\prime \prime}$ & 1.8 \\
\hline 28 Jun, 2006 & 3000 & UVES & BLUE346, RED580 & $1.0^{\prime \prime}$ & & 40970,42310 & 077.A-0714(A) & $0.5^{\prime \prime}$ & 1.0 \\
\hline 27 Jul, 2006 & 3000 & UVES & BLUE437, RED760 & $1.0^{\prime \prime}$ & & 40970,42310 & 077.A-0714(A) & $1.5^{\prime \prime}$ & 1.0 \\
\hline 15 Jul, 2013 & $3028^{a}$ & X-Shooter & UVB, VIS, NIR & $1.3^{\prime \prime}, 1.2^{\prime \prime}, 1.2^{\prime \prime}$ & -14.2 & $4000,6700,3890$ & 091.A-0246(A) & $0.7^{\prime \prime}$ & 1.0 \\
\hline \multirow[t]{2}{*}{$31 \mathrm{Jul}, 2013$} & 3028 & X-Shooter & UVB, VIS, NIR & $1.3^{\prime \prime}, 1.2^{\prime \prime}, 1.2^{\prime \prime}$ & -72.1 & $4000,6700,3890$ & 091.A-0246(A) & $1.0^{\prime \prime}$ & 1.0 \\
\hline & 3028 & X-Shooter & UVB, VIS, NIR & $1.3^{\prime \prime}, 1.2^{\prime \prime}, 1.2^{\prime \prime}$ & 29.2 & $4000,6700,3890$ & 091.A-0246(A) & $1.0^{\prime \prime}$ & 1.0 \\
\hline \multirow{2}{*}{03 Aug, 2013} & 3028 & X-Shooter & UVB, VIS, NIR & $1.3^{\prime \prime}, 1.2^{\prime \prime}, 1.2^{\prime \prime}$ & -76.3 & $4000,6700,3890$ & 091.A-0246(A) & $0.9^{\prime \prime}$ & 1.0 \\
\hline & 3028 & X-Shooter & UVB, VIS, NIR & $1.3^{\prime \prime}, 1.2^{\prime \prime}, 1.2^{\prime \prime}$ & -5.2 & $4000,6700,3890$ & 091.A-0246(A) & $0.9^{\prime \prime}$ & 1.0 \\
\hline 31 Aug, 2013 & 3028 & X-Shooter & UVB, VIS, NIR & $1.3^{\prime \prime}, 1.2^{\prime \prime}, 1.2^{\prime \prime}$ & -85.5 & $4000,6700,3890$ & 091.A-0246(A) & $0.8^{\prime \prime}$ & 1.0 \\
\hline
\end{tabular}

$a$ The exposure time for all X-Shooter data is 3000 (UVB), 3028 (VIS), and 960×3 (NIR) sec. PA convention is East-of-North. The UVES data were obtained with the slit rotating on the sky to follow parallactic angle, so the PA was changing during the exposure.

observations were made in "STARE mode" because the position of the sub-DLA galaxy on sky was unknown. Indeed, the "STARE mode" helps to avoid the quasar counter-image to fall on top of the galaxy, but the absence of telescope nodding then limits the quality of the NIR arm. The slit widths were $1.3^{\prime \prime}, 1.2^{\prime \prime}$, and $1.2^{\prime \prime}$ in the UVB, VIS, and NIR arms respectively. The expected resolving power with the above setup, and for seeing matching the slits, is 4000, 6700 and 3890 in the UVB, VIS and NIR arms, respectively. Those resolutions are computed from the slit widths, assuming that the slit is matched to the seeing. However, since we were searching for line emission from a source at an unknown position we used slits wider than the seeing, and the actual point source resolutions, based on the median seeing of $0.9^{\prime \prime}$, are 5330,8930 , and 5190. We requested the observations to be performed in service mode but erroneously set the rotator angle to parallactic at the start of each observations. Fortunately, the PAs cover the regions around the QSO fairly well (see Fig. 11) and we detect the source in the spectra with PA $-14^{\circ}$ and $-5^{\circ}$ (east of north).

The X-Shooter spectra are reduced with the X-Shooter pipeline 2.4.0 (Modigliani et al. 2010). The standard procedures within STARE mode for master bias, master dark, flat-fielding, wavelength calibration, and the 2D maps for rectification of the spectra are performed. The flux standard stars EG 274 (on 15 July and 31 August 2013) and Feige 110 (on 31 July and 03 August 2013) are observed and used to flux calibrate the science spectra of the corresponding nights. The resulting flux-calibration is not absolute, but rather removes the instrumental effects from the quasar continuum.

The spectra were corrected for galactic extinction $\left(E_{B-V}=0.019\right)$ using the extinction maps of Schlegel et al. (1998). In Fig. 2 we present the section around the DLA of the extracted $2 \mathrm{D}$ spectra at $\mathrm{PAs}=-14^{\circ}$ and $-5^{\circ}$ (top two panels) and the final combined 1D QSO spectrum (bottom panel). We clearly detect the Ly $\alpha$ emission in both spectra (the dark blobs above the 2D QSO spectrum). The Ly $\alpha$ emission is well separated from the QSO spectrum and is therefore not present into the 1D QSO spectrum.
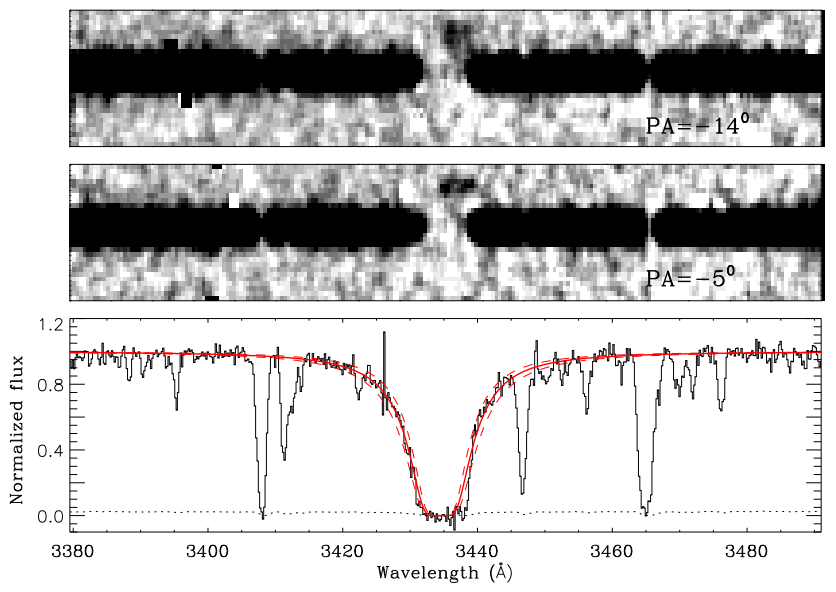

Figure 2. The sub-DLA emission and absorption. The upper two panels show the $2 \mathrm{D}$ spectra from the PAs $-14^{\circ}$ and $-5^{\circ}$. The Ly $\alpha$ emission from the sub-DLA-galaxy counterpart is seen close to the red wing of its absorption line, offset by $2.4^{\prime \prime}$ upwards from the quasar spectrum. In the lower panel we show the combined, extracted 1D spectrum of the QSO and its corresponding noise spectrum (dotted line). The red solid and dashed lines show the best fit H I column density profile $(z=1.825, \log N(\mathrm{H} \mathrm{I})=19.78 \pm$ $0.11 \mathrm{~cm}^{-2}$ ) and its $1 \sigma$ uncertainty, respectively.

\section{RESULTS}

\subsection{Gas absorption properties and metallicity}

Zafar et al. (2013b) reported an H i column density of the sub-DLA of $\log N(\mathrm{H} \mathrm{I})=19.84 \pm 0.14 \mathrm{~cm}^{-2}$. The X-Shooter data is also used to derive the $\mathrm{H}$ I column density and the estimated column density is $\log N(\mathrm{H} \mathrm{I})=19.78 \pm 0.11 \mathrm{~cm}^{-2}$ (Fig. 2), consistent with the UVES results within $1 \sigma$.

The high-resolution UVES spectrum allows us to perform absorption line profile fitting and determine column densities from lines that are not heavily saturated. The column densities have been derived using the Voigt profile fitting, $\chi^{2}$ minimisation routine FITLYMAN within the MIDAS environment (Fontana \& Ballester 1995). Laboratory wavelengths and oscillator strengths are taken from Morton 
Table 2. Metallicity with respect to solar of the sub-DLA.

\begin{tabular}{|c|c|c|c|}
\hline $\mathrm{X}$ & $\begin{array}{c}\log N_{X} \\
\mathrm{~cm}^{-2}\end{array}$ & {$[\mathrm{X} / \mathrm{H}]$} & {$[\mathrm{X} / \mathrm{H}]_{\mathrm{tot}}^{a}$} \\
\hline $\mathrm{H} \mathrm{I}_{\mathrm{I}}$ & $19.84 \pm 0.14$ & $\cdots$ & $\cdots$ \\
\hline $\mathrm{N}_{\mathrm{I}}$ & $<14.71$ & $<-0.96$ & $\cdots$ \\
\hline Zn II & $<12.30$ & $<-0.10$ & $\cdots$ \\
\hline Si II & $14.68 \pm 0.06$ & $-0.67 \pm 0.15$ & $\cdots$ \\
\hline O I & $>16.08$ & $>-0.45$ & $\cdots$ \\
\hline C II & $>15.97$ & $>-0.30$ & $\cdots$ \\
\hline Fe II & $14.11 \pm 0.04$ & $-1.23 \pm 0.15$ & $\cdots$ \\
\hline $\mathrm{Al}$ III & $12.87 \pm 0.07$ & $\cdots$ & $\cdots$ \\
\hline $\mathrm{Al}$ II & $>13.61$ & $>-0.68$ & $>-0.61$ \\
\hline Cr II & $<12.79$ & $<-0.69$ & $\cdots$ \\
\hline Mg II & $>14.86$ & $>-0.58$ & $>-0.58$ \\
\hline $\mathrm{Mg} \mathrm{I}$ & $<12.96$ & $\cdots$ & $\cdots$ \\
\hline
\end{tabular}

$a$ Total abundances from the sum of all listed

ionisation states.

(2003). The global fit returns the best fit parameters for the central wavelength, column density and Doppler turbulent broadening $(b)$, as well as $1 \sigma$ errors on each quantity.

Absorption lines detected from Si II, Fe II, and Al III display a multicomponent structure covering $\sim 80 \mathrm{~km} \mathrm{~s}^{-1}$ around $z_{\mathrm{abs}}=1.825$. A first velocity profile estimation is manually derived for Fe II 2260, 1608, 2374 and $2344 \AA$. Seven components are used to model the observed absorption lines. This approach forces FITLYMAN to converge towards the optimal solution. Si II 1808 and $1304 \AA$ lines are added, maintaining the redshifts and doppler parameters previously derived, thereby providing initial solution for the Si II column density. Thus, the software is run again fully using these parameters as first guest solutions. We finally added Al III 1862 and perform again the previous operation. The resulting doppler parameter for the third component $\left(b=1.2 \mathrm{~km} \mathrm{~s}^{-1}\right)$ is so small that it might be unphysical. For this reason, a value of $b=3 \mathrm{~km} \mathrm{~s}^{-1}$ is enforced and the fit is performed again. The simultaneous multiple Voigt profile fitting is shown in Fig. 3. For saturated and non-detected lines, we report $3 \sigma$ column density lower and upper limits respectively (see Table 2). The overall measured, i.e. before considering a possible ionisation correction, sub-DLA metallicity is found to be $[\mathrm{Si} / \mathrm{H}]=-0.67 \pm 0.15$.

As a consistency check we compare our absorption line results with the X-Shooter data. We measure rest equivalent widths of unsaturated lines and find $W_{r}^{\lambda 1808}=0.02 \pm 0.03 \AA$, $W_{r}^{\lambda 2374}=0.16 \pm 0.04 \AA$, and $W_{r}^{\lambda 1862}=0.08 \pm 0.03 \AA$, for Si II, FeII, and AlıII respectively. Using those equivalent widths, and assuming the lines to be on the linear part of the curve-of-growth, we determine column densities log $N$ (Si II) $=14.48 \pm 0.19 \mathrm{~cm}^{-2}, \log N(\mathrm{Fe}$ II $)=14.01 \pm 0.10 \mathrm{~cm}^{-2}$, and $\log N(\mathrm{Al} \mathrm{III})=12.97 \pm 0.14 \mathrm{~cm}^{-2}$ in full agreement with the UVES data.

The error on the $[\mathrm{Si} / \mathrm{H}]$ metallicity given above includes only the observational errors. It is well known that for subDLAs there may be a large range of possible ionisation levels, which would cause additional uncertainty on the metallicity unless the ionisation parameter can be constrained. While the gas in sub-DLAs is found to range from predominantly ionised to predominantly neutral (Meiring et al. 2007, 2009; Lehner et al. 2014; Fumagalli et al. 2016), the relative ionisation correction varies from element to element (e.g. $<0.2$ dex for Fe II). Our [Si/H] metallicity could therefore be affected by ionisation effects, by dust depletion, or the combination of both. The measured [Si/Fe] ratio indeed indicates that at least one of these two effects is at play, if not both of them.

Unfortunately we have, in the present case, only a limit on the Al III/Al II ratio, which may not be a reliable indicator of the ionisation parameter because the recombination coefficient of Al II is likely overestimated Nussbaumer \& Storev 1986). Our best constraint is therefore that of $[\mathrm{O} / \mathrm{H}]>-0.45$ which is independent of ionisation effects for this $\mathrm{H}$ I column density, and we will simply rely on this throughout the paper. Because $\alpha$-elements may be enhanced by up to a factor of two, the lower limit on the actual metallicity is -0.75 (3 $\sigma)$.

\subsection{Emission lines}

The detection of Ly $\alpha$ in emission above the position of the QSO is clear from Fig. 2. The impact parameter is large enough that the faint Ly $\alpha$ emission does not show up in the extracted QSO spectrum, but there is a small overlap which would leave a residual of the bright QSO in the DLA galaxy spectrum if not corrected for. In order, therefore, to extract the $1 \mathrm{D}$ spectrum of the $\mathrm{Ly} \alpha$ emission line we must first spectral point spread function (SPSF) subtract the QSO 2D spectrum in the overlapping region (Møller 2000). Because the seeing is different in the two exposures, we perform the SPSF subtraction on the two spectra individually, then add them. The resulting 2D frame is seen in the lower panel of Fig. 4 (boxcar smoothed for presentation purposes).

From the non-smoothed, summed, and SPSF subtracted $2 \mathrm{D}$ spectrum we then extract the $1 \mathrm{D}$ spectrum shown in the top panel of Fig. 6. It is seen that all Ly $\alpha$ emission is to the right (high redshift) side of the absorber, i.e. that the profile matches the typical high redshift profile with a blue cut-off and a red tail (e.g., Francis et al. 1996; Hu et al. 2010; Castro-Tirado et al. 2010; Noterdaeme et al. 2014). However, in this case it is also seen that the profile appears to be double peaked. In the figure we have therefore fitted the profile with a double gaussian, and find that it is fitted well by two gaussian profiles (shown red in overlay) centred on 3435.9 and $3438.6 \AA$ with measured FWHM of 1.7 and $2.1 \AA$ respectively. After correction for resolution the intrinsic FWHM is found to be 1.5 and $1.9 \AA$, corresponding to $130 \mathrm{~km} \mathrm{~s}^{-1}$ and $165 \mathrm{~km} \mathrm{~s}^{-1}$. The separation between the two peaks corresponds to $230 \pm 40 \mathrm{~km} \mathrm{~s}^{-1}$. The two peaks are seen to be clearly separated also in the stacked $2 \mathrm{D}$ spectrum.

The measured flux of the Ly $\alpha$ emission line in the two spectra is $F_{\mathrm{Ly} \alpha}=5.1 \pm 0.8 \times 10^{-18} \mathrm{erg} \mathrm{s}^{-1} \mathrm{~cm}^{-2} \AA^{-1}$ and $6.2 \pm 0.8 \times 10^{-18} \mathrm{erg} \mathrm{s}^{-1} \mathrm{~cm}^{-2} \AA^{-1}$ for $\mathrm{PA}=-14^{\circ}$ and $-5^{\circ}$, respectively. The two measurements are fully consistent with each other, but they obviously only include the flux passing through the slit in each case. Different slit PA and different seeing causes the slit loss to be different for the two observations, so part of the difference may reflect the two setups. Together the two measurements provide a $10 \sigma$ detection of the $\operatorname{Ly} \alpha$ emission. We convert the Ly $\alpha$ luminosity density to SFR using the relation from Kennicutt (1998): $\mathrm{SFR}\left(\mathrm{M}_{\odot} \mathrm{yr}^{-1}\right)=7.9 \times \mathrm{L}(\mathrm{H} \alpha)\left(\mathrm{erg} \mathrm{s}^{-1}\right)$ and case B recombination theory for flux ratio conversion: $\mathrm{Ly} \alpha / \mathrm{H} \alpha=8.7$. We 


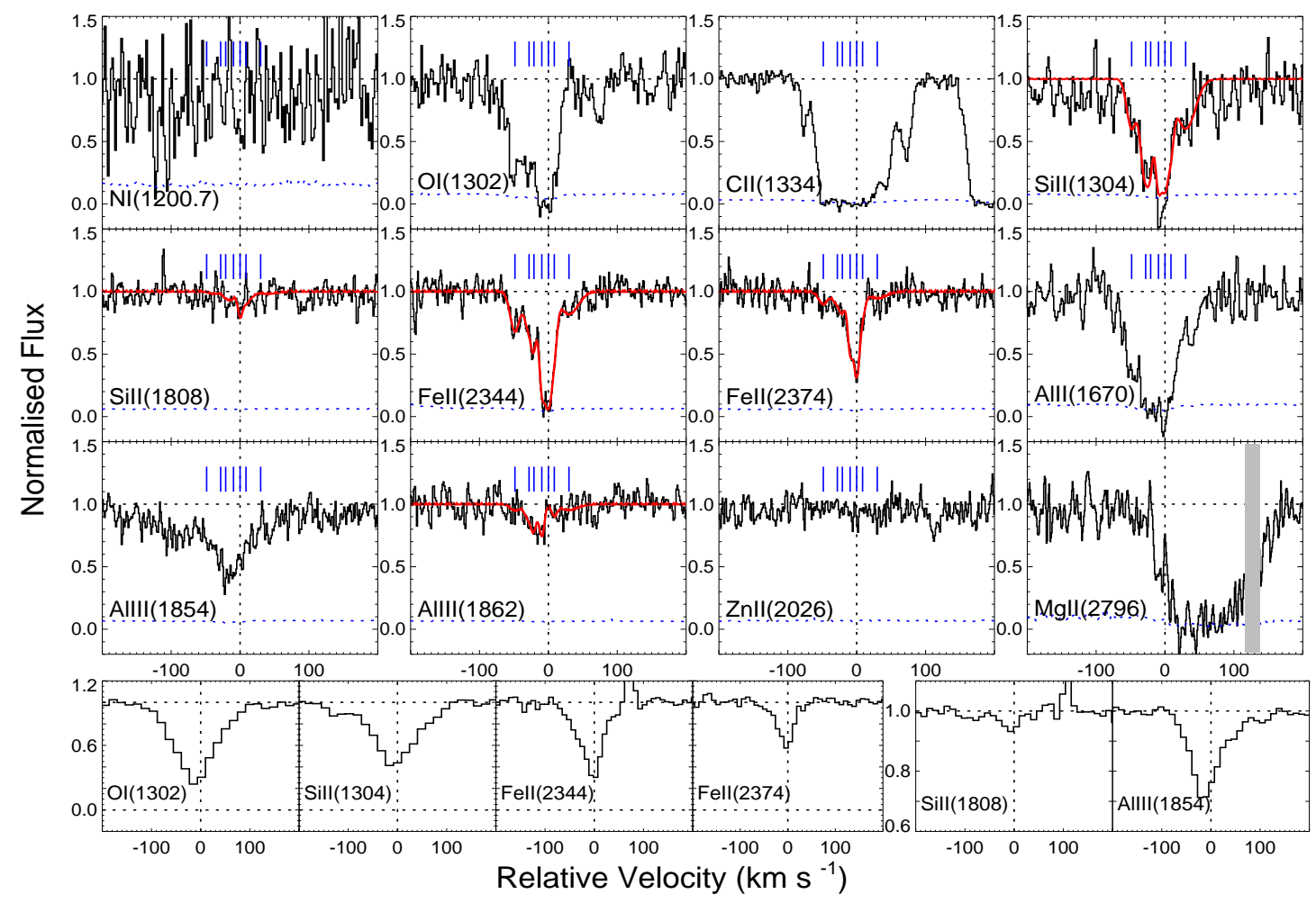

Figure 3. Voigt-profile fits (red overlay) of metal lines for the sub-DLA at $z_{\mathrm{abs}}=1.82516$ (zero velocity) in Q 2239-2949. Normalised quasar spectrum and error spectrum are shown in black and blue dotted lines, respectively. The blue tick marks indicate the locations of the velocity components. The grey shaded area marks contamination from a telluric absorption line. The top three rows present the UVES data and fits, the bottom row presents some of the same lines from the X-Shooter spectrum as useful comparison. For instance, it is seen that the depression of AlIII (1854 $\AA$ ) at around $-100 \mathrm{~km} \mathrm{~s}^{-1}$ in the UVES spectrum is not seen in the X-Shooter spectrum so it is probably not real. All the other lines are fully consistent in both spectra.

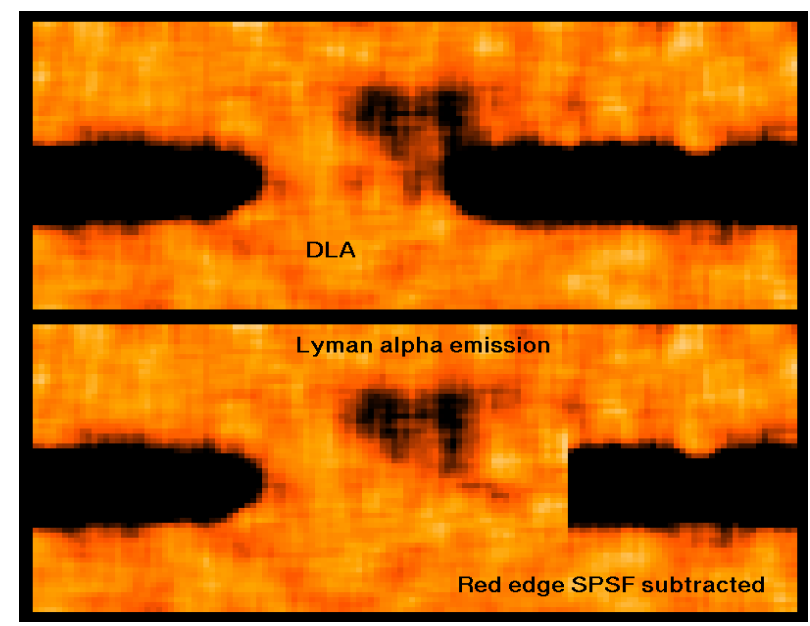

Figure 4. Stack of Ly $\alpha$ emission from the sub-DLA observed at $\mathrm{PA}=-14^{\circ}$ and $-5^{\circ}$. The top panel shows the direct stack, while in the lower panel each spectrum was SPSF subtracted at the red side of the DLA line prior to the summing. There is evidence that the Ly $\alpha$ emission has two peaks.

find that the measured fluxes correspond to a dust uncorrected $\mathrm{SFR}_{\mathrm{Ly} \alpha}$ of $0.13 \pm 0.02 \mathrm{M}_{\odot} \mathrm{yr}^{-1}$. The true value is likely to be somewhat higher due to unknown slit loss corrections. We estimate (based on slit width and seeing) that this correction is less than a factor of two, i.e. $\mathrm{SFR}_{\mathrm{Ly} \alpha}$ is in the range $0.11-0.26 \mathrm{M}_{\odot} \mathrm{yr}^{-1}$.

We searched the spectrum for other emission lines and found marginal evidence for [O II] line emission in the NIR spectrum at the same impact parameter as for $\operatorname{Ly} \alpha\left(2.4^{\prime \prime}\right)$ and is roughly centred on the sub-DLA absorption lines, contrary to the $\operatorname{Ly} \alpha$ emission. Also as for $\operatorname{Ly} \alpha$ the line is found to be stronger and better detected at $\mathrm{PA}=-5^{\circ}$ than at $\mathrm{PA}$ $=-14^{\circ}$. In Fig. 5 we show the sum of the two spectra. The [O II] doublet is partly hidden behind the strong residuals left from the subtraction of an airglow line. For presentation purposes, in Fig. 5 we have smoothed the spectrum along the dispersion direction and masked out the residuals of two strong airglow lines. The $1 \mathrm{D}$ spectrum of the [O II] line is shown in the lower panel of Fig. [6]

\subsection{Gas kinematics}

We have detected Ly $\alpha$ and [O II] emission from the host galaxy of the sub-DLA system, providing us with an opportunity to study the gas kinematics of the galaxy probed at two different locations. In Fig. 6 we summarise the kinematic information of all the gas components, plotting them in velocity relative to a fiducial redshift chosen to be $z=1.82516$. It is seen that H I and the low-ion metal lines are lined up at the same central redshift in the absorption sightline, but also that the [O II] emitting gas some $20.8 \mathrm{kpc}$ away has the same 


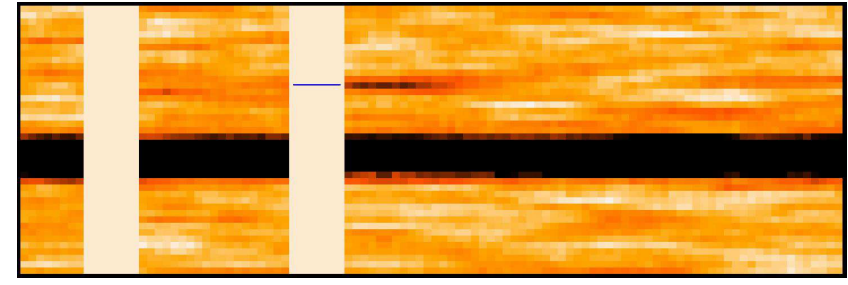

Figure 5. Section of the $2 \mathrm{D}$ spectrum (sum of $\mathrm{PA}=-5^{\circ}$ and $-14^{\circ}$ spectra) around the expected wavelength of the [O II] doublet. The spectrum has been smoothed along the dispersion direction to enhance the visibility of the [O II] line which is seen as a dark feature above the quasar spectrum. The residuals from the subtraction of two strong air glow lines have been masked out (vertical bands) and the horizontal blue line in the rightmost of those marks the $2.4^{\prime \prime}$ impact parameter of the Ly $\alpha$ emission for comparison.

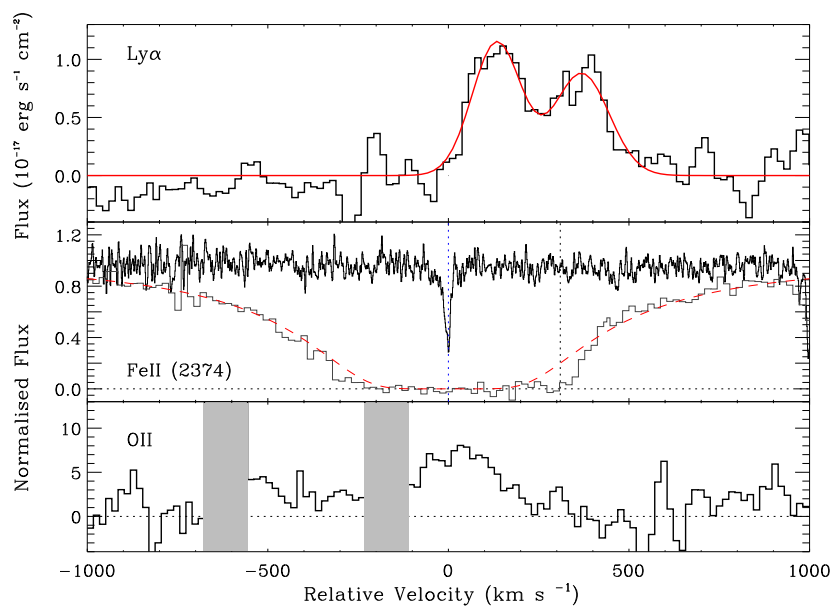

Figure 6. Velocities of absorption and emission components relative to $z=1.82516$. In the middle panel the $\mathrm{H}$ I absorption profile is overlaid on the un-saturated profile of a Fe II absorption line. Note the additional absorption component at $v \approx 300 \mathrm{~km} \mathrm{~s}^{-1}$ in the $\mathrm{H}$ I absorption profile (black vertical dotted line). In the top panel we show the double peaked profile of the Ly $\alpha$ emission from the galaxy (black) with a fit of two gaussian profiles (red). The bottom panel shows the extracted spectrum at the expected position of the $[\mathrm{O}$ II] doublet (grey shaded areas mark positions of masked air glow lines). A marginal detection is achieved.

redshift. We measure a central wavelength of the [O II] doublet of $10533.9 \AA \pm 1.5$ corresponding to a relative velocity of $v=10 \pm 42 \mathrm{~km} \mathrm{~s}^{-1}$. In effect this means that the neutral sub-DLA gas is at rest (radially) with respect to the galaxy, whereas the Mg II absorbing gas extends out to $+170 \mathrm{~km} \mathrm{~s}^{-1}$ in radial velocity (Fig. 3). I.e. where the narrow line subDLA phase appears to be at rest inside the extended halo, at least part of the $\mathrm{Mg}$ II absorbing gas belongs to a more turbulent component.

In contrast to the relative velocity agreement for [O II], the Ly $\alpha$ emission has a complex "double hump" structure with both peaks offset towards higher redshifts. Double peaked Ly $\alpha$ emission has been reported in relation to DLAs in the past (e.g., Fynbo et al. 2010; Zafar et al. 2011; Noterdaeme et al. 2012), but in the form of two peaks straddling a central absorption feature at the systemic redshift.
Verhamme et al. (2008), analysed Ly $\alpha$ emission lines in a sample of 11 high redshift galaxies from the FORS deep field. To obtain high enough signal-to-noise for their analysis they were restricted to include only the intrinsically brightest galaxies, and only those with the largest Ly $\alpha$ equivalent widths. All 11 galaxies feature the sharp blue cut-off of the Ly $\alpha$ emission, as well as the tail towards the red. Interestingly two of the galaxies also show a secondary hump well to the red of the first peak and at about $+300 \mathrm{~km} \mathrm{~s}^{-1}$ with respect to the galaxy redshift. In that work this was taken as evidence for gas outflow, but the same line structure could simply be the result of superposition of two single lines, i.e. two individual Ly $\alpha$ emitting clouds, at slightly different velocities.

In the case of Q2239-2949 the secondary peak is offset by $+400 \mathrm{~km} \mathrm{~s}^{-1}$, and we note that on the red side of the sub-DLA trough an additional absorption feature is seen at $\approx 300 \mathrm{~km} \mathrm{~s}^{-1}$. This is most likely a Ly $\alpha$ absorption line and could in that case be arising in a separate absorbing cloud in the CGM of the galaxy. In this case it might be related to the second peak of the Ly $\alpha$ emission complex. We do not detect any metal lines associated with the secondary absorption component.

In order to derive the internal kinematics of the absorbing gas, we calculate the absorption line velocity width, $\Delta v_{90}$ (Prochaska \& Wolfe 1997; Ledoux et al. 2006). For this, it is standard practice to carefully select a line (if possible more than one) which is unsaturated, unblended, and of sufficiently high signal-to-noise (for details see e.g Ledoux et al. 2006). An alternative method is to measure $\Delta v_{90}$ on the combine information from several lines of the same transition via the Voigt profile optical depth (VPOD; Quiret et al. 2016). This method also overcomes possible effects from the differential instrumental resolutions, line spread functions and SNRs as well as blending/saturation in the absorption profiles.

In the UVES spectrum of Q 2239-2949 there is no perfect line to measure, and we have therefore adopted the VPOD method and used a combined Voigt profile fit to two lines of FeII. Fig. 7 shows the resulting integrated optical depth for the Fe II ( $2374 \AA$ ) with inferred velocity widths of $\Delta v_{90}(\mathrm{Fe}$ II $)=64 \mathrm{~km} \mathrm{~s}^{-1}$. The X-Shooter data have lower resolution, but higher signal-to-noise, and since we know from the model fit that the Fe II (2374 $\AA$ ) line is unsaturated, we can obtain a direct measurement from this line, and we find $83 \mathrm{~km} \mathrm{~s}^{-1}$. Because of the lower resolution of X-Shooter this value needs to be corrected for resolution, and following the method described in eq(1) of Arabsalmani et al. (2015) we obtain an intrinsic value of $68 \mathrm{~km} \mathrm{~s}^{-1}$. The two methods and the two data sets are therefore in excellent agreement.

\subsection{Impact parameter}

We detect Ly $\alpha$ emission (see Fig. 4) at the $-14^{\circ}$ and $-5^{\circ}$ position angles. We also detect $[\mathrm{O} \mathrm{II}]$ at lower $\mathrm{S} / \mathrm{N}$ (see Fig. 5). Unfortunately, the $\mathrm{H} \alpha, \mathrm{H} \beta$, [N II], and [O III] lines all fall on telluric regions, and are therefore unaccessible. The angular separation between the QSO and the sub-DLA galaxy can be converted to the projected galaxy impact parameter, yielding information about the gaseous extent of the galaxy halo. Using both the Ly $\alpha$ and the [O II] lines we measure the impact parameter between the QSO and the sub-DLA 


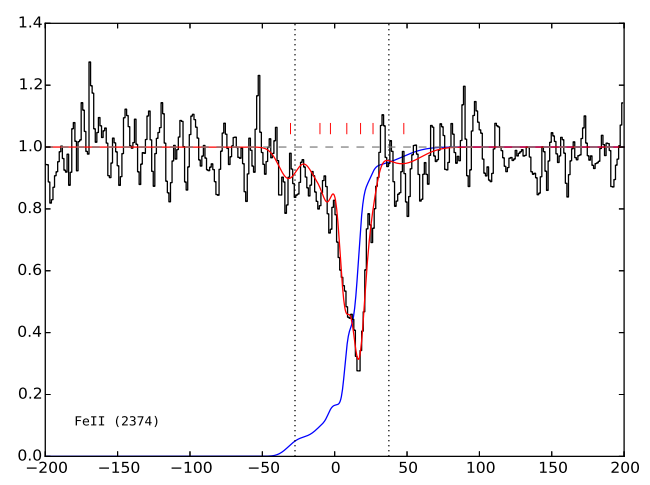

Figure 7. VPOD method Quiret et al. 2016) relying on the Voigt profile fit of multiple transitions of the same ion. The fit is shown here in red, the integrated optical depth in blue and the observed spectrum for the Fe II ( $2374 \AA$ ) transition is shown in black for illustration purpose. This method is therefore independent of blending, saturation, and noise in the spectrum.

galaxy (Fig. 4 \& 5) to be $b=22^{\prime \prime} 4 \pm 0$ '. 2 , corresponding to $20.8 \pm 1.7 \mathrm{kpc}$ at $z=1.825$.

\subsection{Dust properties}

Presence of dust within the absorber will have two effects. First it will result in a depletion of certain metals onto the dust grains, second it could cause a general reddening of the continuum. The non-detections of many elements and the saturation of other lines makes it hard to infer precise dust depletion fractions, instead we are able to set limits on the depletion factor of iron compared to zinc: $[\mathrm{Zn} / \mathrm{Fe}]$ $<1.13$. The value indicate higher amount of depletion with respect to the typical values for DLAs (Noterdaeme et al. 2008; De Cia et al. 2016).

Comparing our X-Shooter spectrum of Q 2239-2949 (scaled to the $2 \mathrm{dF} b$-band photometry) to the quasar template spectrum from Vanden Berk et al. (2001) and Glikman et al. (2006), and using the Small Magellanic Cloud extinction law from Pei $(1992)$, we find no evidence of dust reddening with $E(B-V)<0.02$. However, we stress the limitations of this approach. First, the X-Shooter flux calibration is not absolute and we performed no correction for slit-loss. Given that quasars are known to be variable objects (Palanque-Delabrouille et al. 2015), the rescaling to the $2 \mathrm{dF} b$-band photometry might not totally overcome these drawbacks. Furthermore, intrinsic colours of quasars are also known to vary from one object to another so that a comparison to a quasar template does not directly untangle reddening of the background quasar from reddening of the intervening absorber (Péroux et al. 2014).

\section{SUMMARY AND DISCUSSION}

We have presented new X-Shooter data, as well as previously unpublished archival UVES data, of the $z=1.82516$ intervening sub-DLA absorber towards the quasar Q 22392949. We combined those data with a re-analysis of pre- viously published archival UVES data. Our results can be summarised as follows:

(i) We report the detection of the absorbing galaxy in emission. The galaxy is clearly detected in Ly $\alpha$ at high signal-to-noise and the emission line is resolved. In addition a tentative detection of $[\mathrm{O} \mathrm{II}]$ is reported. However, the sky subtraction residuals in the $\mathrm{X}$-shooter data are significant, and the reality of the $[\mathrm{O} \mathrm{II}]$ line detection requires independent confirmation.

(ii) PA and impact parameter. The detection of the absorbing galaxy was secured using the "slit triangulation" technique. It was detected at slit position angles $\mathrm{PA}=-14^{\circ}$ and $-5^{\circ}$. Using this, together with its position in the slits, we find that the most likely position of the galaxy is at a $\mathrm{PA}(\mathrm{E}$ of $\mathrm{N})$ of $-10^{\circ} \pm 15^{\circ}$, and an impact parameter of $2^{\prime \prime} .4$ $\pm 0^{\prime \prime} .2$, or $20.8 \pm 1.7 \mathrm{kpc}$.

(iii) SFR. We measure a Ly $\alpha$ line flux of $F_{\mathrm{Ly} \alpha}=5.7 \times$ $10^{-18} \mathrm{erg} \mathrm{s}^{-1} \mathrm{~cm}^{-2} \AA^{-1}$, corresponding to a dust uncorrected $\mathrm{SFR}$ of $0.13 \mathrm{M}_{\odot} \mathrm{yr}^{-1}$. Because of the large impact parameter, the uncertainty in the position angle of the emitter, and the unknown physical size of the line emitting region, slit losses are likely to be significant (we estimate up to a factor of two), and the inferred SFR will then be a similar factor higher.

The unknown dust correction could be even more important. It is well known that dust absorption of Ly $\alpha$ photons can cause an additional underestimate of the SFR. An estimate of this effect can be obtained from the observation of both IR continuum and Ly $\alpha$ based SFRs, in a sample of narrow band Ly $\alpha$ selected galaxies out to a redshift of $z=2.3$ (Nilsson \& Møller 2009). From their Figure 3 it is seen that the measured correction ranges from a factor of 1 to $2 \times 10^{3}$. It is also seen that the correction strongly correlates with the reddening, and the largest corrections are all corresponding to super massive ULIRGs. In 3.5 we report evidence for a relatively high amount of dust depletion in this sub-DLA, but we see no direct evidence for reddening of the QSO continuum. We conclude that in this sightline there is some dust present, but not enough to cause a detectable reddening signature. From Nilsson \& Møller (2009) we then conclude that if this sightline is typical for the galaxy, then the SFR dust correction is almost certainly less than a factor of 10 . It is possible that other sightlines through the galaxy may contain more dust, additional data are required to answer this question.

(iv) Kinematics and velocity width. We measure the $\Delta v_{90}$ parameter of the absorption system both directly on the data, and on the fitted Voigt profile model. We find that the two methods give consistent results, and we adopt $\Delta v_{90}=64 \mathrm{~km} \mathrm{~s}^{-1}$ as the best value.

The Ly $\alpha$ emission line displays the typical features of a sharp blue cut-off at the absorption redshift and an extended red tail. In this object the red tail appears to have an additional hump, which is significantly offset $\left(+230 \pm 40 \mathrm{~km} \mathrm{~s}^{-1}\right)$ from the main peak. This second peak may be interpreted in terms of radiation transfer in the case of large scale outflows, but could also simply be related to the Ly $\alpha$ absorption line seen at a similar redshift.

The tentative detection of an [O II] emission line is at a relative velocity of $+10 \pm 40 \mathrm{~km} \mathrm{~s}^{-1}$. From the compilation of relative velocities of [O II] emission in Table 6 of Fvnbo et al. 
(2013), we find that values lie in the range $9-200 \mathrm{~km} \mathrm{~s}^{-1}$ with a median of $38 \mathrm{~km} \mathrm{~s}^{-1}$. This new system is therefore in excellent agreement with previous studies. Since the $[\mathrm{O}$ II] redshift is our best estimator for the systemic redshift, we take this to mean that the absorbing sub-DLA phase kinematics not only internally very narrow, but it also appears to be almost at rest with respect to the galaxy $20.8 \mathrm{kpc}$ away. I.e. the cold CGM at this position does not seem to be wildly turbulent or disturbed.

(v) Metallicity and impact parameter relations. We measure a metallicity of $[\mathrm{O} / \mathrm{H}]>-0.75$ for the subDLA at $z=1.82516$ with $N(\mathrm{HI})=19.84 \pm 0.14 \mathrm{~cm}^{-2}$. A larger impact parameter for lower column density systems has been consistently predicted by numerical simulations for decades (e.g., Katz et al. 1996; Pontzen et al. 2008), and has equally consistently been confirmed by observations (Møller \& Warren 1998; Monier et al. 2009; Rao et al. 2011; Péroux et al. 2011). Similarly, an impact parameter vs metallicity relation was predicted by Møller et al. (2004) and confirmed by Krogager et al. (2012).

Based on observations of several types of high redshift galaxies, Fvnbo et al. (2008) formulated an empirical model to fit DLA galaxies, GRB host galaxies, and Lyman Break Galaxies with a single model. The model has proven useful for predicting and describing the statistical distributions of DLA galaxy scaling relations, and has been confirmed by the current larger statistical sample compiled by Krogager et al. (2012). Comparing our new sub-DLA galaxy to the relations shown in figure 3 of that paper, shows that it falls perfectly on both relations. It seems to be a prototypical sub-DLA galaxy.

(vi) Are sub-DLAs dynamically different from DLAs? Meiring et al. (2007) and Kulkarni et al. (2010) have shown that sub-DLAs have higher metallicity than DLAs when the two systems have the same $\Delta v_{90}$. They suggest that this could mean that sub-DLA galaxies and DLA galaxies are different, and have different masses. The observation could however more simply be understood by stating that, for the same metallicity (i.e. same mass) a subDLA has a narrower absorption line profile than a DLA. In the context of the galaxy we report on here, we confirm that indeed the absorption would be expected to have $\Delta v_{90}>207 \mathrm{kms}^{-1}$ for a DLA (using the optimal fits from Møller et al. (2013) and including the redshift evolution), which is significantly wider than the $64 \mathrm{~km} \mathrm{~s}^{-1}$ we measure. We also find a large impact parameter $(20.8 \mathrm{kpc})$, and further find that the absorber has a very low velocity with respect to the systemic velocity of the galaxy. We take those observations to support the view that sub-DLAs likely belong to the same galaxies as DLAs, but that they are caused by gas in the outskirts of the galaxy halos whereas DLAs belong to gas closer to the galaxy itself, or even to gas inside the galaxy. Support for this also comes from the observations of GRB sightlines which go through the central parts of galaxies and follow the same relations as intervening DLAs (Arabsalmani et al. 2015).

Is this sub-DLA on the Main Sequence of star formation? Based on the observed metallicity lower limit, redshift, and impact parameter, we use the prescription in Christensen et al. (2014) (their equation 3) to determine the corresponding stellar mass limit and find $\log \left(\mathrm{M}_{*} / \mathrm{M}_{\odot}\right)>$
$9.5 \pm 0.4$. We have here adopted the reported scatter (0.39) as the error, but it should be kept in mind that for sub-DLAs the scatter of that relation may be larger (Kulkarni et al. 2010). In 3.2 we found a dust uncorrected SFR of $0.13 \mathrm{M}_{\odot} \mathrm{yr}^{-1}$, but at $z=1.8$ a main sequence galaxy of this mass has a much higher SFR (Whitaker et al. 2014; Kochiashvili et al. 2015). Even applying the maximum correction for dust and slit losses (a factor of 20) it is still about one order of magnitude too low. This indicates that either the true SFR correction is indeed an order of magnitude higher (i.e. there is more dust closer to the centre of the galaxy), or this galaxy has been quenched and is well below the main sequence. An observational determination of both the stellar mass and SFR for this galaxy is possible with current instrumentation, and would settle the issue directly.

\section{ACKNOWLEDGEMENTS}

The research leading to these results has received funding from the European Research Council under the European Union's Seventh Framework Program (FP7/20072013)/ERC Grant agreement no. EGGS-278202. CP and SQ would like to thanks the BINGO! ('history of Baryons: INtergalactic medium/Galaxies cO-evolution') project by the Agence Nationale de la Recherche (ANR) under the allocation ANR-08-BLAN-0316-01. CP thanks the ESO science visitor program for support.

\section{REFERENCES}

Arabsalmani, M., Møller, P., Fynbo, J. P. U., et al. 2015, MNRAS, 446, 990

Ballester, P., Modigliani, A., Boitquin, O., et al. 2000, The Messenger, 101, 31

Bouché, N., Lehnert, M. D., Aguirre, A., Péroux, C., \& Bergeron, J. 2007, MNRAS, 378, 525

Castro-Tirado, A. J., Møller, P., García-Segura, G., et al. 2010, A\&A, 517, A61

Ceverino, D., Sanchez-Almeida, J., Muñoz-Tuñon, C., et al. 2015, arXiv:1509.02051

Christensen, L., Møller, P., Fynbo, J. P. U., \& Zafar, T. 2014, MNRAS, 445, 225

Croom, S. M., Smith, R. J., Boyle, B. J., et al. 2001, MNRAS, 322, L29

De Cia, A., Ledoux, C., Mattsson, L., et al. 2016, arXiv:1608.08621

Dekker, H., D’Odorico, S., Kaufer, A., Delabre, B., \& Kotzlowski, H. 2000, in Society of Photo-Optical Instrumentation Engineers (SPIE) Conference Series, ed. M. Iye \& A. F. Moorwood, Vol. 4008, 534-545

Fontana, A. \& Ballester, P. 1995, The Messenger, 80, 37

Francis, P. J., Woodgate, B. E., Warren, S. J., et al. 1996, ApJ, 457, 490

Fumagalli, M., O'Meara, J. M., \& Prochaska, J. X. 2016, MNRAS, 455, 4100

Fynbo, J. P. U., Geier, S. J., Christensen, L., et al. 2013, MNRAS, 436, 361

Fynbo, J. P. U., Laursen, P., Ledoux, C., et al. 2010, MNRAS, 408, 2128 
Fynbo, J. P. U., Ledoux, C., Noterdaeme, P., et al. 2011, MNRAS, 413, 2481

Fynbo, J. P. U., Prochaska, J. X., Sommer-Larsen, J., Dessauges-Zavadsky, M., \& Møller, P. 2008, ApJ, 683, 321 Glikman, E., Helfand, D. J., \& White, R. L. 2006, ApJ, 640,579

Hu, E. M., Cowie, L. L., Barger, A. J., et al. 2010, ApJ, 725,394

Katz, N., Weinberg, D. H., Hernquist, L., \& MiraldaEscude, J. 1996, ApJL, 457, L57

Kennicutt, Jr., R. C. 1998, ARAA, 36, 189

Kereš, D., Vogelsberger, M., Sijacki, D., Springel, V., \& Hernquist, L. 2012, MNRAS, 425, 2027

Kochiashvili, I., Møller, P., Milvang-Jensen, B., et al. 2015, A\&A, 580, A42

Krogager, J.-K., Fynbo, J. P. U., Ledoux, C., et al. 2013, MNRAS, 433, 3091

Krogager, J.-K., Fynbo, J. P. U., Møller, P., et al. 2012, MNRAS, 424, L1

Krumholz, M. R. 2012, ApJ, 759, 9

Kulkarni, V. P., Khare, P., Som, D., et al. 2010, New Astronomy, 15, 735

Ledoux, C., Petitjean, P., Fynbo, J. P. U., Møller, P., \& Srianand, R. 2006, A\&A, 457, 71

Lehner, N., O'Meara, J. M., Fox, A. J., et al. 2014, ApJ, 788, 119

Meiring, J. D., Lauroesch, J. T., Kulkarni, V. P., et al. 2009, MNRAS, 397, 2037

Meiring, J. D., Lauroesch, J. T., Kulkarni, V. P., et al. 2007, MNRAS, 376, 557

Modigliani, A., Goldoni, P., Royer, F., et al. 2010, in Society of Photo-Optical Instrumentation Engineers (SPIE) Conference Series, Vol. 7737

Møller, P. 2000, The Messenger, 99, 31

Møller, P., Fynbo, J. P. U., \& Fall, S. M. 2004, A\&A, 422, L33

Møller, P., Fynbo, J. P. U., Ledoux, C., \& Nilsson, K. K. 2013, MNRAS, 430, 2680

Møller, P. \& Warren, S. J. 1998, MNRAS, 299, 661

Monier, E. M., Turnshek, D. A., \& Rao, S. 2009, MNRAS, 397, 943

Morton, D. C. 2003, ApJS, 149, 205

Neeleman, M., Wolfe, A. M., Prochaska, J. X., \& Rafelski, M. 2013, ApJ, 769, 54

Nilsson, K. K. \& Møller, P. 2009, A\&A, 508, L21

Noterdaeme, P., Laursen, P., Petitjean, P., et al. 2012, A\&A, 540, A63

Noterdaeme, P., Ledoux, C., Petitjean, P., \& Srianand, R. 2008, A\&A, 481, 327

Noterdaeme, P., Petitjean, P., Ledoux, C., \& Srianand, R. 2009, A\&A, 505, 1087

Noterdaeme, P., Petitjean, P., Pâris, I., et al. 2014, A\&A, 566, A24

Nussbaumer, H. \& Storey, P. J. 1986, A\&AS, 64, 545

Palanque-Delabrouille, N., Magneville, C., Yèche, C., et al. 2015, arXiv:1509.05607

Pei, Y. C. 1992, ApJ, 395, 130

Péroux, C., Bouché, N., Kulkarni, V. P., \& York, D. G. 2013, MNRAS, 436, 2650

Péroux, C., Bouché, N., Kulkarni, V. P., York, D. G., \& Vladilo, G. 2011, MNRAS, 410, 2237
Péroux, C., Bouché, N., Kulkarni, V. P., York, D. G., \& Vladilo, G. 2012, MNRAS, 419, 3060

Péroux, C., Kulkarni, V. P., \& York, D. G. 2014, MNRAS, 437, 3144

Peroux, C., Rahmani, H., Quiret, S., et al. 2016, arXiv:1609.07389

Pontzen, A., Governato, F., Pettini, M., et al. 2008, MNRAS, 390, 1349

Prochaska, J. X. \& Wolfe, A. M. 1997, ApJ, 487, 73

Quiret, S., Péroux, C., Zafar, T., et al. 2016, MNRAS submitted

Rao, S. M., Belfort-Mihalyi, M., Turnshek, D. A., et al. 2011, MNRAS, 416, 1215

Sánchez-Ramírez, R., Ellison, S. L., Prochaska, J. X., et al. 2015, arXiv:1511.05003

Schlegel, D. J., Finkbeiner, D. P., \& Davis, M. 1998, ApJ, 500,525

Shull, J. M., Danforth, C. W., \& Tilton, E. M. 2014, ApJ, 796,49

Vanden Berk, D. E., Richards, G. T., Bauer, A., et al. 2001, AJ, 122, 549

Verhamme, A., Schaerer, D., Atek, H., \& Tapken, C. 2008, A\&A, 491, 89

Vernet, J., Dekker, H., D'Odorico, S., et al. 2011, A\&A, 536, A105

Warren, S. J., Møller, P., Fall, S. M., \& Jakobsen, P. 2001, MNRAS, 326, 759

Weatherley, S. J., Warren, S. J., Møller, P., et al. 2005, MNRAS, 358, 985

Whitaker, K. E., Franx, M., Leja, J., et al. 2014, ApJ, 795, 104

Wolfe, A. M., Turnshek, D. A., Smith, H. E., \& Cohen, R. D. 1986, ApJS, 61, 249

Zafar, T., Møller, P., Ledoux, C., et al. 2011, A\&A, 532, A51

Zafar, T., Péroux, C., Popping, A., et al. 2013a, A\&A, 556, A141

Zafar, T., Popping, A., \& Péroux, C. 2013b, A\&A, 556, A140

This paper has been typeset from a $\mathrm{T}_{\mathrm{E}} \mathrm{X} / \mathrm{L} \mathrm{AT}_{\mathrm{E}} \mathrm{X}$ file prepared by the author. 